\title{
Combined Laser-Based Measurements for Micro- and Nano-Scale Transport Phenomena
}

\author{
Koichi Hishida and Yohei Sato \\ * Corresponding author: Tel.: +81-45-566-1739; Fax: +81-45-566-1720; Email: hishida@sd.keio.ac.jp \\ Department of System Design Engineering, Faculty of Science and Technology, Keio University \\ 3-14-1 Hiyoshi, Kohoku-ku, Yokohama, 223-8522, Japan
}

\begin{abstract}
This paper summarizes our recent works in combined laser-based measurement techniques for investigating micro- and nano-scale transport phenomena. Micron-resolution particle image velocimetry combined with the laser induced fluorescence (LIF) technique has been developed for analyzing velocity and ion concentration distributions simultaneously. The measurement system was based upon a confocal microscope to realize the depth-resolution of approximately $2 \mu \mathrm{m}$, and we have applied this technique to liquid-liquid mixing flows, gas-liquid two-phase flows and gas permeation phenomena through membranes. To evaluate the electrostatic potential at a solid-liquid interface (i.e., zeta-potential), the LIF technique was extended with evanescent wave illumination, and only the fluorescent dye within approximately $100 \mathrm{~nm}$ from a microchannel wall was irradiated. The technique was applied to microdevices with a surface modification pattern, and the zeta-potential distribution was successfully visualized. Two proposed developments will contribute to novel applications related to microscale multiphase flows or electrokinetics.
\end{abstract}

Keywords: Micro-PIV, LIF, Confocal microscopy, Evanescent wave illumination, Zeta-potential

\section{Introduction}

Rapid progress in micro- and nanotechnologies has contributed to the innovation of various industrious applications within electronic engineering, automobile engineering, information and communications technology and so forth. Recently, this technology has allowed the development of microfluidic devices which have been widely used for biological and chemical analysis applications, e.g., lab-on-a-chip and micro total analysis system (micro-TAS) [1, 2]. The devices have microchannels with a width ranging from a few $\mu \mathrm{m}$ to a few hundred $\mu \mathrm{m}$, and have the ability to integrate several operations including transport, mixing and chemical reactions of gas-liquid or liquidliquid flows for realizing the miniaturization of large chemical analytical instruments. In the integrated devices, the flow is controlled by several parameters, including fluid velocities, ion concentration distributions, electric charge of channel wall surface, i.e., a zeta-potential and so on. For further high-accuracy and design optimization of the devices, it is expected that micro- and nanoscale transport phenomena will be investigated by evaluating the above experimental parameters, obtained from the visualization techniques. Previous research into visualization techniques for microchannel flows has been summarized by Sinton [3], and Lindken et al. [4] has reviewed various velocity measurements using micronresolution particle velocimetry (micro-PIV).

This paper summarizes our recent works in the field of laser-based quantitative imaging techniques to investigate micro- and nanoscale transport phenomena. Section 2 describes the experimental approach with a spatial resolution of micron-order [5-11], and the method enables the analysis of velocity and ion concentration distributions to be made simultaneously, because of enhancing the microPIV by combining it with laser induced fluorescence (LIF) using confocal microscopy. Section 3 describes the nanoscale measurement tech-nique, that is LIF with evanescent wave illumination [12-15]. The technique can realize the evaluation of electrostatic potentials at a solid-liquid interface i.e., zeta-potentials. 


\section{Imaging for Microscale Transport Phenomena}

For the quantitative evaluation of transport phenomena in microscale multiphase flows, the imaging technique has been developed to measure the velocity and ion concentration distributions simultaneously [6]. This technique was achieved by combining micronresolution particle imaging velocimetry (micro-PIV) with laser induced fluorescence (LIF). The fluorescent particles with $1 \mu \mathrm{m}$ diameter and fluorescent dye were utilized for the micro-PIV and LIF measurements, respectively, and were added to each aqueous solution at an amount of $5 \times 10^{-4}$ weight/volume and $1 \times 10^{-4} \mathrm{~mol} / \mathrm{l}$ concentration, respectively. Tables 1 and 2 list respectively the properties of particles (Invitrogen Corp.) and dye (Wako Pure Chemical Industries, Ltd., Fluorescein sodium salt) whose fluorescent intensity increases with a decrease in the proton concentration. The important factor is that the excitation wavelength of particles is close to that of the dye, while the emission wavelength of the particles differs from the dyes. Figure 1 illustrates the measurement system developed in this work which is composed of an inverted microscope (Nikon Corp., TE2000), a confocal scanner (Yokogawa Electric Corp., CSU22), a continuous Ar laser, an objective lens (Nikon Corp.) and a 3CCD color camera (Hamamatsu Photonics K. K., C7780-20, $672 \times 512$ pixels, 8 bits $\times 3$ ). This set-up corresponds to a measurement area of $434 \mu \mathrm{m} \times 330 \mu \mathrm{m}$. A laser beam from the continuous $\mathrm{Ar}$ laser operating at a wavelength of $488 \mathrm{~nm}$ and a power of $50 \mathrm{~mW}$ was introduced into the confocal scanner by an optical fiber. The beam illuminated particles and dye in a microchannel through a micro-lens-array disk, a pin-hole-array disk, and an objective lens. The micro-lens-array disk and the pin-holearray disk have respectively 20,000 microlenses and 20,000 pin-holes whose diameter is $50 \mu \mathrm{m}$. Both disks are set up coaxially because of focusing the beam on the corresponding pin-hole. Fluorescence from particles and dye
Table 1. Property of fluorescent particles

\begin{tabular}{lll} 
Material & \multicolumn{3}{c}{ Polystyrene } \\
Mean diameter & {$[\mu \mathrm{m}]$} & 1.0 \\
Density & {$\left[\mathrm{g} / \mathrm{cm}^{3}\right]$} & 1.055 \\
Absorption wavelength & {$[\mathrm{nm}]$} & 488 \\
Emission wavelength & {$[\mathrm{nm}]$} & 645 \\
\hline
\end{tabular}

Table 2. Property of fluorescent dye

\begin{tabular}{lcc} 
Material & Fluorescein sodium salt \\
Molecular weight & [g/mol] 376.28 \\
Absorption wavelength & {$[\mathrm{nm}]$} & 494 \\
Emission wavelength & {$[\mathrm{nm}]$} & 518 \\
\hline
\end{tabular}

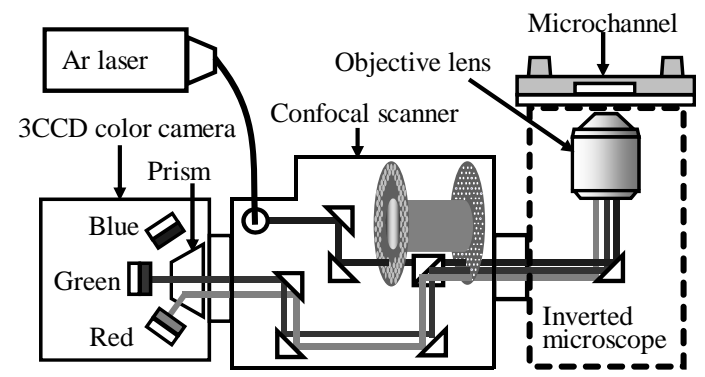

Figure 1. Schematic of measurement system using confocal microscope for investigating microscale transport phenomena.

passes through the optical path of the measurement system in the reverse direction and reaches the pin-hole-array disk. The infocus fluorescence with wavelengths longer than $520 \mathrm{~nm}$ is reflected by a dichroic mirror located between the two disks and captured by the 3CCD camera, while the out-of-focus fluorescence is removed by the pin-holes. The depth resolution of the confocal microscope (known as the optical slice thickness), $\Delta z_{O S T}$, is calculated from following equation

$\Delta z_{\text {OST }}=\sqrt{\left(\frac{0.88 \lambda_{e m}}{n-\sqrt{n^{2}-N A^{2}}}\right)^{2}+\left(\frac{\sqrt{2} n \cdot P D}{N A}\right)^{2}}$,

where $\lambda_{e m}$ is the emission wavelength, $n$ is a refractive index of the fluid between the microchannel and the objective lens, $N A$ is the numerical aperture of the objective lens and $P D$ is the modified pin-hole diameter that shows $2.5 \mu \mathrm{m}$. The optical slice thickness of the present system results in $5.0 \mu \mathrm{m}$ for an air immersion 20× magnification objective lens 
with a numerical aperture of 0.75 and $2.2 \mu \mathrm{m}$ for an oil immersion 40× magnification objective lens with a numerical aperture of 1.3, which give a higher depth resolution, compared with the conventional epifluorescent microscope. Furthermore, the 3CCD camera has the ability to separate RGB colors by an internal prism, and the spectra of blue, green and red sensors are approximately $400-490 \mathrm{~nm}, 490-580 \mathrm{~nm}$ and $580-660$ $\mathrm{nm}$, respectively. It is obvious from tables 1 and 2 that the green and red sensors capture the fluorescence of dye and particles, respectively. This fact indicates that the present system enables the separation of the fluorescent information of the particles from that of the dye and measures the velocity and ion concentration distribution simultaneously. The advantage of this work is in having a $t$ simple optical system composed of only one laser and one camera, while the conventional system would require two lasers and two cameras for PIV and LIF.

This imaging approach has been applied to the liquid-liquid mixing and a chemical reacting flow [6], liquid flow in a surface modified microchannel [7], solid-liquid twophase flow [8, 9] and gas-liquid two-phase flow [10, 11]. Figure 2 shows the velocityvectors and proton concentration distribution of the chemical reacting flow formed by merging buffer solutions with $\mathrm{pH} 6.0$ and 7.7 in the junction area of the T-shaped microchannel [6]. Both streams were moved towards the junction area at the same flow rate by a pressure-driven flow, for a Reynolds number of $2.41 \times 10^{-2}$ based on the hydraulic diameter of $89 \mu \mathrm{m}$ and the bulk velocity of $272 \mu \mathrm{m} / \mathrm{s}$. The arrows in figure 2 show the velocity-vectors and the contour map indicates the proton distribution. The vectors are directed towards the liquid-liquid interface $(y$ $=200 \mu \mathrm{m}$ ) from each inlet and the $\mathrm{pH}$ gradient over the range of $\mathrm{pH} 6.6-7.7$ in the spanwise direction ( $Y$-direction) was formed by merging the two aqueous solutions. This result enables us to evaluate the convection and diffusion expressed in the local scalar transport equation which will contribute to the enhancement of mixing and chemical reaction in microfluidic

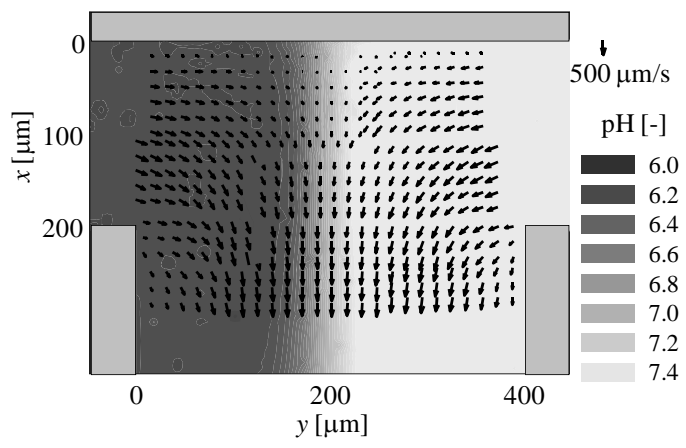

Figure 2. Velocity-vector and $\mathrm{pH}$ distribution of the liquid-liquid mixing flow field with the chemical reaction.
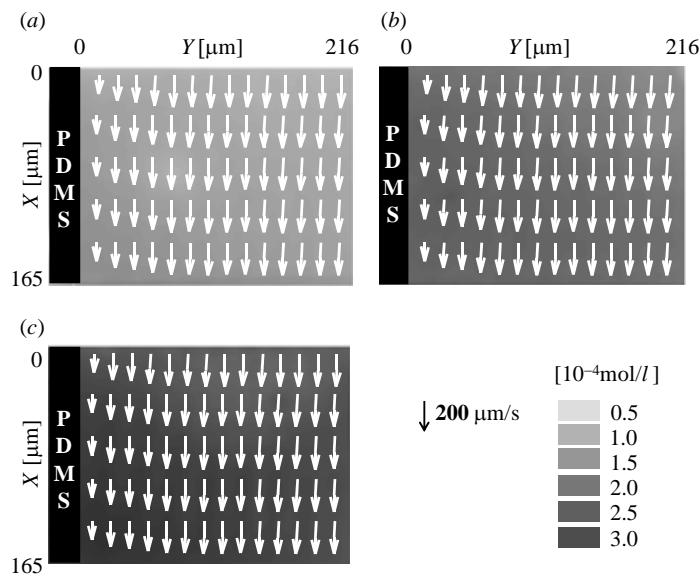

Figure 3. Velocity-vector and $\mathrm{CO}_{2}$ concentration distributions of liquid flow field in which gas carbon dioxide dissolved through a polymer membrane. The experiments used microfluidic devices with surface roughness of (a) $200 \mathrm{~nm}$, (b) $300 \mathrm{~nm}$ and (c) $400 \mathrm{~nm}$.

devices. Furthermore, figure 3 exhibits the velocity-vectors and carbon dioxide concentration distributions in microfluidic devices with gas permeability achieved through a polymer membrane [11]. In the devices, microchannels were manufactured by using cryogenic micro machining which has the potential to change the surface roughness [16]. The increase in its surface roughness yields the surface area. Figure 4 summarizes the relationship between the carbon dioxide concentration and the surface area of the membranes, and surface roughnesses of $\mathrm{Ra}$ $200 \mathrm{~nm}, 300 \mathrm{~nm}$ and $400 \mathrm{~nm}$ correspond to surface areas of $1.40 \times 10^{-9} \mathrm{~m}^{2}, 1.71 \times 10^{-9} \mathrm{~m}^{2}$ and $1.98 \times 10^{-9} \mathrm{~m}^{2}$, respectively. It is clear that 
the increase in the surface area gives an increase in the gas dissolution, and the carbon dioxide concentration dissolved through the membranes has a linear dependence with the surface area. This fact indicates that the fabrication process is effective for the performance of a multiphase flow system in microfluidic devices.

\section{Imaging for Nanoscale Transport Phenomena}

One of the important characteristics of the micro- and nanoscale transport phenomena is that the properties of a material surface, i.e., its electric charge, chemical affinity and wettability, exert a large effect because the surface-to-volume ratio increases as the dimensions decrease. These surface effects yield electrokinetic flows, specific adsorption of macromolecules and parallel multi-phase flows, and can provide various microfluidic applications as components for miniaturized systems. Therefore our group has developed laser based measurement techniques for nearwall transport phenomena. Here, our fluorescence imaging method using evanescent wave illumination (nano-LIF) is described [12, 13]. This technique has been applied to the quantitative visualization of a surface electrostatic potential, i.e., the zeta-potential, which is dominant for electrokinetic flows and electric adsorption.

The near-wall flow imaging is achieved by employing the evanescent wave generated by total internal reflection of light, as illustrated in figure 5. The evanescent wave penetrates into the working fluid to distances of the order of $100 \mathrm{~nm}$. The measurement principle for the zeta-potential is based upon an electrostatic interaction between fluorescence ions and the charged surface. Fluorescent cations (red) and fluorescence anions (green), dichlorotris (1, 10-phenanthroline) ruthenium (II) hydrate (divalent cation, $50 \mu \mathrm{mol} / \mathrm{l}$, absorption: 449 nm, emission: $582 \mathrm{~nm}$ ), and Alexa Fluor 488 (divalent anion, $5 \mu \mathrm{mol} / \mathrm{l}$, absorption: $494 \mathrm{~nm}$, emission: $519 \mathrm{~nm}$ ), were used as fluorescent probes. The fluorescence ions were dissolved

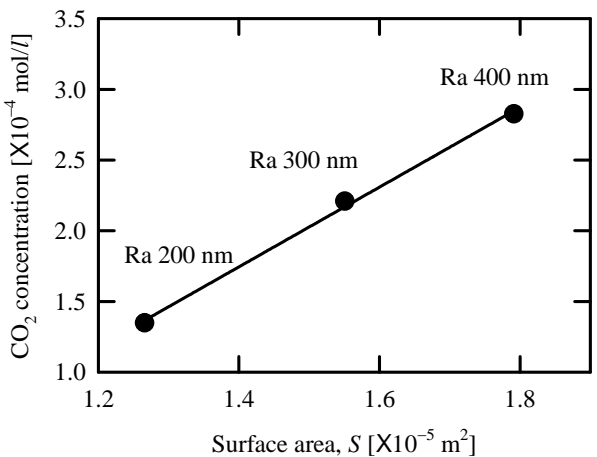

Figure 4. Relationship between $\mathrm{CO}_{2}$ concentration and surface area of membrane calculated from surface roughness.

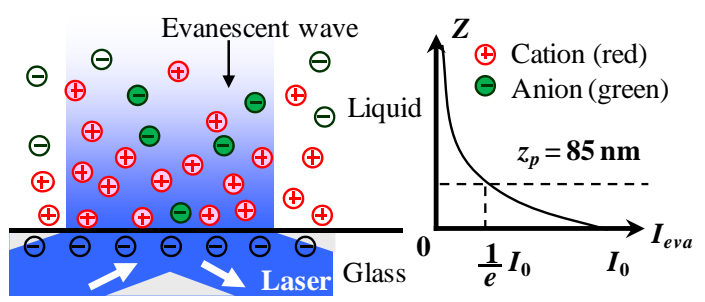

Figure 5. Schematic showing fluorescent ions illuminated by the evanescent wave that decays exponentially from the wall ( $I_{\text {eva }}$ : evanescent wave intensity, $I_{0}$ : intensity at interface).

into the working fluid at a negligibly low concentration compared to the electrolyte. The near-wall ions are distributed by the electrostatic forces related to the zetapotential: cations are attracted to the surface and anions are repelled, when the surface is negatively charged. Therefore, the zetapotetnial, $\zeta$, can be estimated from the measured red to green fluorescence intensity ratio, $I_{r} / I_{g}$, which determines the observed color:

$\frac{I_{r}}{I_{g}}=\frac{I_{\text {eva }} \phi_{r} \gamma_{r} c_{r}(\zeta)}{I_{\text {eva }} \phi_{g} \gamma_{g} c_{g}(\zeta)}=f(\zeta)$

where $\phi$ is the quantum yield, $\gamma$ is the molar absorption coefficient, $c$ is the concentration, and $f(\zeta)$ is a function determined by the zetapotential.

The experimental system consisted of a microscope, a $488 \mathrm{~nm}$ wavelength laser (Coherent Inc., Sapphire 488-30), an objective 

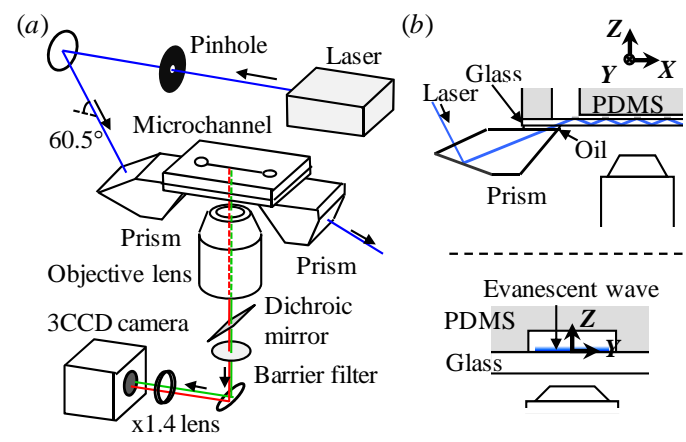

Figure 6. Schematics of $(a)$ the measurement system and $(b)$ evanescent wave illumination in a microchannel.

lens (Nikon Corp., CFI Plan Fluor, $\times 20, N A=$ 0.45 ), and the 3CCD camera (figure 6). A microchannel (width: $4 \mathrm{~mm}$, depth: $50 \mu \mathrm{m}$ ) located on two prisms with index-matching oil is fabricated from PDMS and sealed with a 1mm-thick glass slide. The laser beam is introduced into the glass of the microchannel through the first prism, guided by total internal reflection, and emitted from the other prism. The evanescent wave is generated at the glassliquid interface with a penetration depth of $z_{p}=$ $85 \mathrm{~nm}$. The fluorescence is detected by the 3CCD camera. Prior to the measurements, a relationship between the fluorescent intensity ratio and the zeta-potential represented as $f(\zeta)$ in equation (2) was determined.

The proposed method has been demonstrated by evaluating the surface modification pattern generated by self assembled monolayers (SAMs) on a glass surface. Octadecyltrichlorosilane (OTS) was modified on fused silica by microcontact printing to reduce a negative charge of the wall surface with a tile pattern with a width of 100 $\mu \mathrm{m}$. Using an optical profiler (Zygo Corp., NewView 6200), the thickness of an OTS monolayer was evaluated to be $3-4 \mathrm{~nm}$. The fluorescent solution with $2.5 \mathrm{mmol} / \mathrm{l} \mathrm{NaCl}$ was injected into the microchannel and the measurement was conducted. The tile pattern was visualized in the captured image, as shown in figure $7(b)$. Areas of bare and OTSmodified fused silica could be distinguished by the color of the fluorescence. The cations, which emitted red fluorescence, were attracted (a)
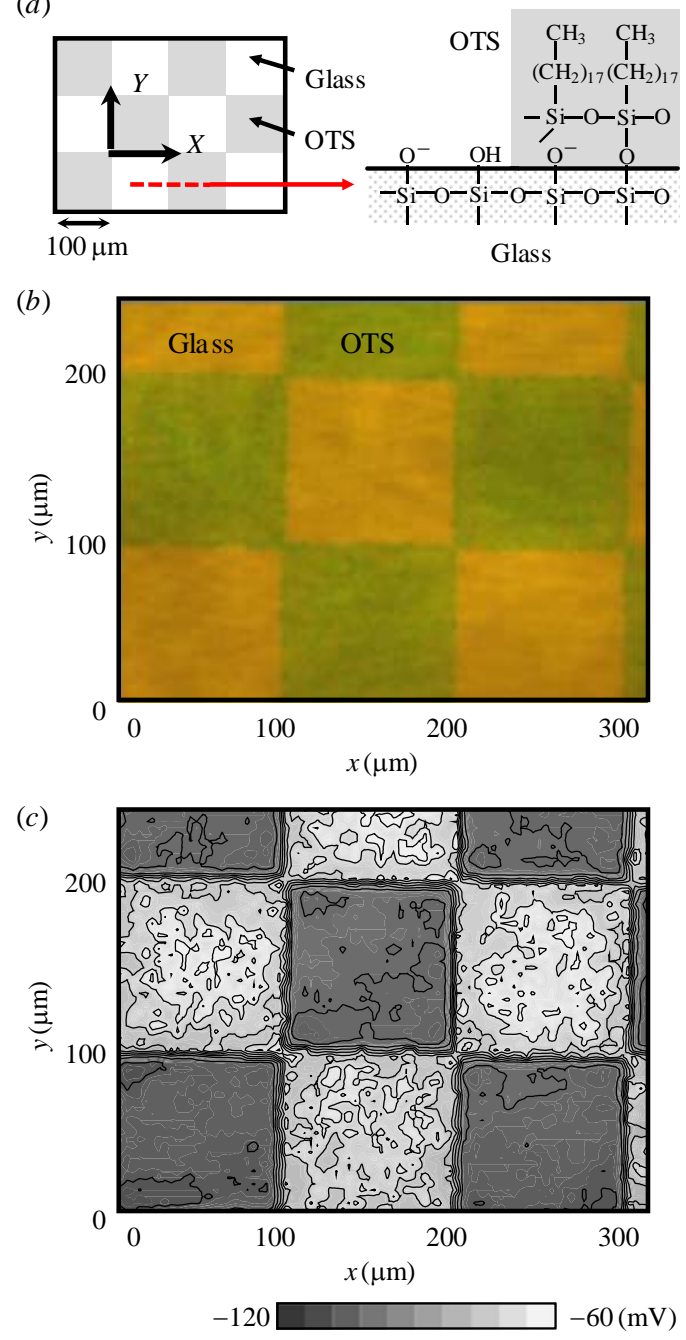

Figure 7. (a) Schematic of the OTS modified surface with a $100 \mu \mathrm{m}$-tile pattern. OTS reduces a negative charge of fused silica surface by exchanging a potion of the chemical end groups of Si-OH with $\left(-\mathrm{CH}_{3}\right)$. (b) A fluorescence image captured by the 3CCD camera. The red and green areas indicate the bare and OTS-modified fused silica, respectively. (c) The zeta-potential distribution by the surface modification. The spatial resolution was determined by the pixel size of the camera and the magnification of the optical system.

to bare glass with a more negative zetapotential resulting in the orange fluorescence, whereas, the image of the OTS modified glass has green fluorescence. Using the image, the zeta-potential was quantitatively evaluated at a high spatial resolution of $0.46 \mu \mathrm{m} \times 0.46 \mu \mathrm{m}$, as shown in figure $7(c)$. It is considered that the zeta-potential on the OTS-modified areas 
varies due to the nonuniformity of the OTS layer, in contrast to the almost uniform zetapotential on fused silica. We have verified our proposed imaging by velocity measurement of electroosmotic flow induced by external electric fields, where the flow pattern is dependent on the zeta-potential distribution. This approach is expected to contribute to further developments in surface modification techniques and miniaturized flow systems.

\section{Conclusions}

This paper introduces the laser-based measurement techniques for micro- and nanoscale transport phenomena established by the authors' group. Information on velocity of the fluid, concentration of ions, and the surface electrostatic potential, is the dominant parameter in the governing equations of fluid dynamics, physics and chemistry, and can be obtained by using fluorescent tracers. We are confident currently that our "integrated measurement” approach will certainly lead to further development of miniaturized flow systems for chemical and biological analysis. However, the individual techniques still have the problem that the data is under the influences of tracer behavior, i.e., diffusion, adsorption and shift of chemical equilibrium, owing to the increased surface-to-volume ratio. Our ultimate goal in future work is to establish a novel nonintrusive flow imaging technique without any tracer materials. It is anticipated that our ongoing research will reveal unknown phenomena hindered by the tracer effects.

\section{References}

[1] Reyes DR, Iossifidis D, Auroux PA, Manz A (2002) Micro total analysis systems. 1. Introduction, theory, and technology. Anal Chem 74: pp.2623-2636.

[2] Auroux PA, Iossifidis D, Reyes DR, Manz A (2002) Micro total analysis systems. 2. Analytical standard operations and applications. Anal Chem 74: pp.2637-2652.

[3] Sinton D (2004) Microscale flow visualization. Microfluid Nanofluid 1: pp.2-21.

[4] Lindken R, Rossi M, Große S, Westerweel J
(2009) Micro-particle image velocimetry ( $\mu$ PIV): Recent developments, applications, and guidelines. Lab on a chip 9: pp.2551-2567.

[5] Ichiyanagi M, Sato Y, Hishida K (2005) Evaluation of electroosmotic velocity and zetapotential in microchannel using submicron fluorescent particles. Trans Jpn Soc Mech Eng 71709: pp.2316-2323.

[6] Ichiyanagi M, Sato Y, Hishida K (2007) Optically sliced measurement of velocity and $\mathrm{pH}$ distribution in microchannel. Exp Fluids 43: pp.425-435.

[7] Ichiyanagi M, Sasaki S, Sato Y, Hishida K (2009) Micro-PIV/LIF measurements on electrokinetically-driven flow in surface modified microchannel. $J$ Micromech Microeng 19: pp.045021(9pp).

[8] Ichiyanagi M, Nakanosono H, Sato Y, Hishida K (2009) Phase separation technique for suspended particles in microchannel utilizing bilayered acoustic fields. J Fluid Sci Technol 4-1: pp.1-12.

[9] Hishida K, Ichiyanagi M, Sato Y (2009) Phase separation technique in two-phase microchannel flow. J Phys Conf Ser 147: pp.012056(14pp).

[10] Tsutsui I, Senga Y, Sato Y (2008) Optical measurements of chemical reaction by gas dissolution in a gas-liquid microchannel flow. 14th Int Symp Appl Laser Tech Fluid Mech (Lisbon, Portugal).

[11] Ichiyanagi M, Sakai K, Kidani S, Kakinuma Y, Sato Y, Hishida K (2011) Evaluation of gas permeability in microfluidic device by confocal micro-PIV combined with LIF technique. ASME/JSME 8th Thermal Engineering Joint Conference (Hawaii, Submitted).

[12] Kazoe Y, Sato Y (2007) Effect of ion motion on zeta-potential distribution at microchannel wall obtained from nanoscale laser-induced fluorescence. Anal Chem 79: pp.6727-6733.

[13] Kazoe Y, Miyakawa S, Miki N, Sato Y (2009) Fluorescence imaging technique of surface electrostatic potential using evanescent wave illumination. Appl Phys Lett 95: pp.234104(3pp).

[14] Kazoe Y, Nakamura T, Sato Y (2010) Evanescentwave/volume illuminated velocity measurements of transient electrokinetically driven flow with nonuniform wall electrostatic potential. Meas Sci Technol 21: pp.055401(10pp).

[15] Senga Y, Nakamura T, Fukumura H, Ichiyanagi M, Sato Y (2010) Near-wall motion of caged fluorescent dye in microchannel flows obtained from evanescent wave molecular tagging. J Fluid Sci Technol 5-2: pp.192-206.

[16] Kakinuma Y, Yasuda N, Aoyama T (2008) Micromachining of soft polymer material applying cryogenic cooling. J Adv Mech Design System Manufacturing 2: pp.560-569. 